\title{
Flutter Solution by Use of Eigenvalues and Eigenvectors of a Complex General Matrix
}

\author{
Ju Qiu \\ P. O. Box 310, School of Aeronautics, Northwestern Polytechnical University \\ Xi'an, 710072, China \\ E-mail: qiuxiaoju@mail.nwpu.edu.cn \\ Qin Sun \\ P. O. Box 310, School of Aeronautics, Northwestern Polytechnical University \\ Xi'an, 710072, China \\ E-mail: sunqin@ nwpu.edu.cn
}

\begin{abstract}
Flutter equations in frequency domain, is solved by use of solutions of eigenvalues and eigenvectors of a real matrix in a state-space form. However, using a complex general matrix to compute eigenvalues and eigenvectors directly analyzes the flutter frequencies and flutter speeds. Furthermore, eigenvalues and eigenvectors of a complex general matrix are derived in detail. Finally, according to the solution principle of eigenvalues and eigenvectors of a complex general matrix, a practical flutter problem is solved through P-K method.
\end{abstract}

Keywords: Flutter Equation, Complex Matrix, Eigenvalue, Eigenvector, Technique for Eigenvalue Tracking

\section{Introduction}

Eigenvalue and eigenvector computation may be the most prolific for special case numerical computation. A numerical solution of characteristic values and vectors for a general real matrix is demonstrated in many books. As a general complex matrix, however, its solution is more intricate, when we consider the size and speed of modern computers.

In general, complex eigenvalues have applications in the solution of some physics problems and some practical engineering problems. For example, some flutter analysis in aircraft design uses eigenvalues in this paper.

\section{Eigenvalues of a General Complex Matrix}

Computing the characteristic equation is usually not a good way to compute eigenvalues for $\mathrm{n}$ greater than 4 or 5 . It becomes difficult to compute the coefficients of the characteristic equation accurately and it is also difficult to compute the roots accurately.

In the present study, in order to save some time, the general complex matrix is first balanced. Unitary similarity transformations are used to reduce this balanced matrix to a complex upper Hessenberg matrix. The QR algorithm is used to compute the eigenvalues of this Hessenberg matrix, which is simplified to the first-order blocks or the second-order blocks. Then, the eigenvectors of the original matrix are computed by the inverse power method.

\subsection{Hessenberg Transform of a Complex Matrix[1][2]}

Let a $n \times n$ complex matrix $A=\left\lfloor a_{i j}\right\rfloor$ be represented in the form $A=\left[a_{1}, a_{2}, \cdots, a_{n}\right]$, where $a_{1}, a_{2}, \cdots, a_{n}$ are vectors of order $n$.

Step1. Suppose that $\mathrm{a}_{11}$ is equal to zero, and the first column of $\mathrm{A}$ is expressed as $\tilde{a}_{1}=\left[0, a_{21}, \cdots, a_{n 1}\right]^{T}$.Unitary transform of image matrix (Householder transform) $\mathrm{Q}_{1}$ is simplified in the form $\left[0, \alpha_{1}, 0, \cdots, 0\right]^{T}$. Let $Q_{1}=I-2 u_{1} u_{1}{ }^{T}$, where I denotes the unit matrix, $u_{1}=\frac{1}{\rho_{1}}\left(\tilde{a} \pm \alpha_{1} e_{2}\right), \alpha_{1}= \pm \sqrt{\sum_{i=2}^{n}\left|a_{i 1}\right|^{2}}, \rho_{1}=\| \tilde{a_{1} \pm \alpha_{1} e_{2} \|_{2}}=\sqrt{2} \sqrt{\alpha_{1}^{2} \pm \alpha_{1} \tilde{a}_{1}{ }^{T} e_{2}}$, and $\rho_{1}$ is defined by 


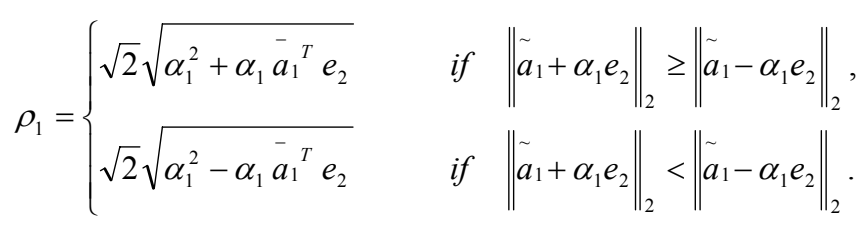

Step2. Let $Q_{1}=\left[\begin{array}{cc}1 & 0 \\ 0 & \mathrm{U}_{1}\end{array}\right]$, and $A=\left[\begin{array}{cc}a_{11} & \mathrm{~W}_{1} \\ \tilde{\mathrm{a}_{1}} & \mathrm{~B}_{1}\end{array}\right]$, where $\tilde{a}_{1}=\left[a_{21}, a_{31}, \cdots, a_{n 1}\right]^{T} \cdot \mathrm{Q}_{1}$ is a unitary similar transform matrix, then

$$
Q_{1}^{-1} A Q_{1}=\left[\begin{array}{ll}
a_{11} & \mathrm{~W}_{1} \mathrm{U}_{1} \\
\mathrm{U}_{1} \tilde{a}_{1} & \mathrm{U}_{1} B_{1} U_{1}
\end{array}\right]=\left[\begin{array}{ll}
a_{11} & \mathrm{~W}_{1} \mathrm{U}_{1} \\
\alpha & \\
0 & \\
\vdots & \mathrm{U}_{1} B_{1} U_{1} \\
0 &
\end{array}\right] \equiv\left[\begin{array}{lll}
a_{11} & \tilde{a}_{12} & \\
& & \mathrm{~W}_{2} \\
\alpha_{1} & \tilde{a}_{22} & \\
0 & & B_{2}
\end{array}\right]
$$

where $\mathrm{B}_{2}$ is a unitary square matrix of size $(\mathrm{n}-2) \times(\mathrm{n}-2)$. Again, let $Q_{2}=\left[\begin{array}{cc}I_{2} & 0 \\ 0 & \mathrm{U}_{2}\end{array}\right]$, then

$$
Q_{2}^{-1}\left(Q_{1}^{-1} A Q_{1}\right) Q_{2}=\left[\begin{array}{llll}
a_{11} & \tilde{\mathrm{a}}_{12} & \\
& & \mathrm{~W}_{2} U_{2} \\
\alpha_{1} & \tilde{\mathrm{a}}_{22} & \\
0 & \mathrm{U}_{2} \tilde{\mathrm{a}}_{2} & \mathrm{U}_{2} B_{2} U_{2}
\end{array}\right]=\left[\begin{array}{lll}
a_{11} & \tilde{\mathrm{a}}_{12} & \\
& & \mathrm{~W}_{2} U_{2} \\
\alpha_{1} & \tilde{\mathrm{a}}_{22} & \\
0 & \alpha_{2} & \\
0 & 0 & \\
\vdots & \vdots & \mathrm{U}_{2} B_{2} U_{2} \\
0 & 0 &
\end{array}\right]
$$

Step3.Similarly, let $Q_{k}=I-2 u_{k} u_{k}{ }^{T}$, where $\alpha_{k}= \pm \sqrt{\sum_{i=k+1}^{n}\left|\tilde{a}_{i k}\right|^{2}}, u_{k}=\frac{1}{\rho_{k}}\left(\tilde{a}_{k} \pm \alpha_{k} e_{k+1}\right)$,

$\rho_{k}=\left\|\tilde{a_{k}} \pm \alpha_{k} e_{k+1}\right\|_{2}=\sqrt{2} \sqrt{\alpha_{k}^{2} \pm \alpha_{k} \tilde{a}_{k}^{T} e_{k+1}}$, and $\rho_{k}$ is defined by

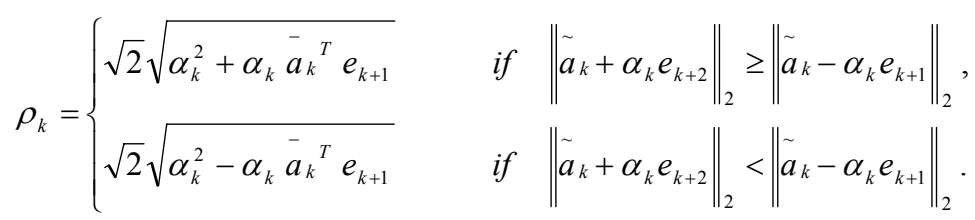

Where $k=1,2, \cdots, n-2$. The unitary similar transform of the matrix A is represented by

$\tilde{Q}_{n-2}^{-1} A \tilde{Q}_{n-2} \equiv Q_{n-2}^{T} \cdots Q_{1}^{T} A Q_{1} \cdots Q_{n-2} \equiv H$, where $\mathrm{H}$ denotes the complex upper Hessenberg matrix, and $\alpha_{1}, \alpha_{2}, \cdots, \alpha_{n-2}$ is the bisub-diagonal elements of this Hessenberg matrix.

\subsection{Hessenberg Transform of a Complex Matrix[1][2]}

Suppose that the matrix A is complex square of size $n \times n$, and $\mathrm{A}$ is expressed by

$A=\left[\begin{array}{llll}a_{11} & \mathrm{a}_{12} & \cdots & \mathrm{a}_{1 \mathrm{n}} \\ a_{21} & \mathrm{a}_{22} & \cdots & \mathrm{a}_{2 \mathrm{n}} \\ \vdots & \ddots & & \\ a_{n 1} & \mathrm{a}_{\mathrm{n} 2} & \cdots & \mathrm{a}_{\mathrm{nn}}\end{array}\right]$. The Application of the two step QR algorithm is presented as follows:

1) Let a unitary matrix $\mathrm{Q}_{0}, Q_{0}=\left[\begin{array}{ll}Q_{0}^{(0)} & \mathrm{O} \\ \mathrm{O} & \mathrm{I}_{\mathrm{n}-3}\end{array}\right]$, where $Q_{0}^{(0)}$ is a unitary square matrix of size $3 \times 3$. The unitary similarity transformation can be given by $A_{1}=Q_{0} A Q_{0}$. Let 


$$
\begin{aligned}
& \alpha=a_{n-1, n-1}+a_{n n}, \quad \beta=a_{n-1, n-1} a_{n n}-a_{n-1, n} a_{n, n-1}, \quad p_{0}=a_{11}\left(a_{11}-\alpha\right)+a_{12} a_{21}+\beta, \\
& q_{0}=a_{21}\left(a_{11}+a_{22}-\alpha\right), \quad r_{0}=a_{21} a_{32} . \text { Here }
\end{aligned}
$$

$$
Q_{0}^{(0)}=\left[\begin{array}{ccc}
-\frac{p_{0}}{s_{0}} & -\frac{\mathrm{q}_{0}}{\mathrm{~s}_{0}} & -\frac{\mathrm{r}_{0}}{\mathrm{~s}_{0}} \\
-\frac{\mathrm{q}_{0}}{\mathrm{~s}_{0}} & \frac{\mathrm{p}_{0}}{\mathrm{~s}_{0}}+\frac{r_{0}^{2}}{s_{0}\left(p_{0}+s_{0}\right)} & -\frac{\mathrm{q}_{0} r_{0}}{s_{0}\left(p_{0}+s_{0}\right)} \\
-\frac{r_{0}}{s_{0}} & -\frac{\mathrm{q}_{0} r_{0}}{s_{0}\left(p_{0}+s_{0}\right)} & \frac{\mathrm{p}_{0}}{\mathrm{~s}_{0}}+\frac{q_{0}^{2}}{s_{0}\left(p_{0}+s_{0}\right)}
\end{array}\right]
$$

Where $s_{0}= \begin{cases}\sqrt{\left|p_{0}\right|^{2}+\left|q_{0}\right|^{2}+\left|r_{0}\right|^{2}} & \text { if } \quad\left|p_{0}+s_{0}\right| \geq\left|p_{0}-s_{0}\right| \\ -\sqrt{\left|p_{0}\right|^{2}+\left|q_{0}\right|^{2}+\left|r_{0}\right|^{2}} & \text { if } \quad\left|p_{0}+s_{0}\right|<\left|p_{0}-s_{0}\right|\end{cases}$

2) Similarly, the unitary matrices $Q_{1}, Q_{2}, \cdots, Q_{n-2}$ are defined by

$Q_{i}=\left[\begin{array}{ccc}I_{i} & \mathrm{O} & \mathrm{O} \\ O & \mathrm{Q}_{\mathrm{i}}^{(0)} & \mathrm{O} \\ O & \mathrm{O} & \mathrm{I}_{\mathrm{n}-\mathrm{i}-3}\end{array}\right]$, where $i=1,2, \cdots, n-2, Q_{i}^{(0)}$ is a unitary square matrix of size $3 \times 3$. Let $p_{i}=a_{i+1, i}^{(i)}$, $q_{i}=a_{i+2, i}^{(i)}, \quad r_{i}=a_{i+3, i}^{(i)}$, if $p_{n-2}=a_{n-1, n-2}^{(n-2)}, q_{n-2}=a_{n, n-2}^{(n-2)}$, then $r_{n-2}=0 \cdot Q_{i}^{(0)}$ can be expressed by

$$
Q_{i}^{(0)}=\left[\begin{array}{ccc}
-\frac{p_{i}}{s_{i}} & -\frac{\mathrm{q}_{\mathrm{i}}}{\mathrm{s}_{\mathrm{i}}} & -\frac{\mathrm{r}_{\mathrm{i}}}{\mathrm{s}_{\mathrm{i}}} \\
-\frac{\mathrm{q}_{\mathrm{i}}}{\mathrm{s}_{\mathrm{i}}} & \frac{\mathrm{p}_{\mathrm{i}}}{\mathrm{s}_{\mathrm{i}}}+\frac{r_{i}^{2}}{s_{i}\left(p_{i}+s_{i}\right)} & -\frac{\mathrm{q}_{\mathrm{i}} r_{i}}{s_{i}\left(p_{i}+s_{i}\right)} \\
-\frac{r_{i}}{s_{i}} & -\frac{\mathrm{q}_{\mathrm{i}} r_{i}}{s_{i}\left(p_{i}+s_{i}\right)} & \frac{\mathrm{p}_{\mathrm{i}}}{\mathrm{s}_{\mathrm{i}}}+\frac{q_{i}^{2}}{s_{i}\left(p_{i}+s_{i}\right)}
\end{array}\right]
$$

Where

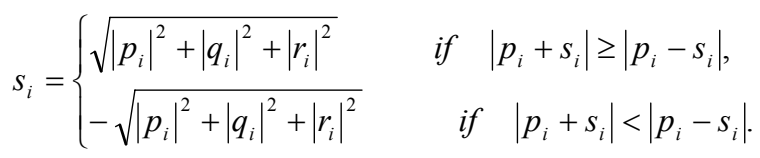

Clearly, the unitary similar transformation can be written as

$A_{i+1}=Q_{i} A_{i} Q_{i}, \quad$ where $i=1,2, \cdots, n-2$.

3) Repeat the above two steps, until this Hessenberg matrix is simplified to the first-order blocks or the second-order blocks in the diagonal blocks. Finally, each first-order block or second-order block is directly computed to the corresponding eigenvalues.

\section{Eigenvectors of a General Complex Matrix[2]}

Assume that the $\mathrm{n} \times \mathrm{n}$ complex matrix A has $\mathrm{n}$ eigenvalues $\lambda_{1}, \lambda_{2}, \ldots, \lambda_{\mathrm{n}}$. Consider the eigenvalue $\lambda_{\mathrm{i}}$, and suppose that a $\mathrm{n} \times \mathrm{n}$ complex matrix $B_{i}=A-\bar{\lambda}_{i} I$, for each $i=1,2, \cdots, n$ has the eigenvalues $\lambda_{1}-\bar{\lambda}_{i}, \lambda_{2}-\bar{\lambda}_{i}, \cdots, \lambda_{n}-\bar{\lambda}_{i}$.

Select $\bar{\lambda}_{i}$, which satisfies $\left|\lambda_{i}-\bar{\lambda}_{i}\right| \leq 10^{-6}$. Apply inverse power method to find the eigenvector $\mathrm{V}_{\mathrm{i}}$ of the smallest $\left|\lambda_{i}-\bar{\lambda}_{i}\right|$ of $\mathrm{Bi}$, that is, the eigenvalue $\lambda_{\mathrm{i}}$ of the matrix A with the corresponding eigenvector. Thus, $\mathrm{V}_{1}, \mathrm{~V}_{2}, \ldots, \mathrm{V}_{\mathrm{n}}$ are all eigenvalues of the matrix A.

\section{A Test Case}

\subsection{Flutter Equation}

Aeroelastic flutter, is a rapid self-feeding motion, potentially destructive, excited by aerodynamic forces, in aircraft structures, control surfaces and so on. Therefore, it is very critical for airplane engineers to analyze aeroelastic flutter.

The fundamental equation for modal flutter analysis by the PK-method[4][5]

$$
\left[p^{2} \bar{M}-p(\rho b V / 4) Q_{I} / k+\bar{K}-\left(\rho V^{2} / 2\right) Q_{R}\right] q=0
$$


Where $p$ is the eigenvalue $\omega(\gamma \pm i), \gamma$ is transient decay rate coefficient, $\rho$ is air density, $V$ is the free-stream velocity, and $q$ are the generalized displacements for a structure with $\bar{M}$ and $\bar{K}$ being the inertial and stiffness properties, respectively. The generalized aerodynamic properties are given by matrix $Q=Q_{R}+i Q_{I}$, where $i=\sqrt{-1}$.

Eq. (6) is usually rewritten in a state-space form:

$$
(A-p I) \bar{q}=0, \text { where } A=\left[\begin{array}{lc}
0 & \mathrm{I} \\
-\dot{\mathrm{M}}^{-1}\left(\bar{K}-\frac{1}{2} \rho V^{2} Q_{R}\right) & \frac{1}{4} \rho V b Q_{I} \bar{M}^{-1} / k
\end{array}\right]
$$

As seen from the above matrix A, the solution of the flutter equation is changed to the eigenvalue problem of the real matrix.

However, let $A^{\prime}=-\bar{M}^{-1} \bar{K}+\frac{1}{2} \rho V^{2} \bar{M}^{-1}\left(Q_{R}+i Q_{I}\right)$, and computing the eigenvalues of the complex matrix A' also solves
the flutter problem.

\subsection{Use of Eigenvector for Eigenvalue Tracking[5]}

In the present study, the solution for one mode at all speeds is found before the solution for the next mode is started. The explanation which follows is for the solution of mode $\mathrm{i}$ only. Although only the solution for mode $\mathrm{i}$ is valid (because the aerodynamic matrix is a function of reduced frequency), the eigenvalues and eigenvectors corresponding to all the other modes are calculated.

The natural frequency of mode $\mathrm{i}$ is used in the first calculation of the reduced frequency at the first speed. The aerodynamic matrix is interpolated and the eigenvalue routine is called to solve the eigenvalues and eigenvectors. The eigenvectors are compared to unit vectors, corresponding to the natural modes, in order to determine which eigenvalue corresponds to mode $\mathrm{i}$. This eigenvalue is used in the next calculation of the reduced frequency and the eigenvalue routine is called again. The process is repeated until the reduced frequency converges. At each successive speed, the converged eigenvalue of the previous speed is used in the first calculation of the reduced frequency. The aerodynamic matrix is interpolated and the eigenvalue routine is called to solve the eigenvalues and eigenvectors. The eigenvectors are compared to the converged eigenvectors of the previous speed to determine which eigenvalue corresponds to mode i. This eigenvalue is used in the next calculation of the reduced frequency.

Eigenvectors are compared as follows: A matrix of scalar products of the converged eigenvectors of the previous speed and the new eigenvectors is calculated. Each column of the matrix corresponds to a new eigenvector and each row to an old eigenvector. The element in row $\mathrm{i}$ and column $\mathrm{j}$ of the matrix is the scalar product of old eigenvector $\mathrm{i}$ and new eigenvector $\mathrm{j}$. The matrix is then searched for the largest element. The corresponding old and new eigenvectors are taken to belong to the same mode.

The scalar product of two complex vectors must be defined to be independent of scaling and phase. A definition which satisfies these conditions is

$$
X \cdot Y=\frac{\sqrt{S_{1}^{2}+S_{2}^{2}}}{\sqrt{S_{3} S_{4}}}
$$

Where

$$
\begin{gathered}
X=\left[X_{1}, X_{2}, \cdots, X_{n}\right]^{T} \\
Y=\left[Y_{1}, Y_{2}, \cdots, Y_{n}\right]^{T} \\
S_{1}=\sum_{i=1}^{n} \operatorname{Re}\left(X_{i}\right) \operatorname{Re}\left(Y_{i}\right)+\operatorname{Im}\left(X_{i}\right) \operatorname{Im}\left(Y_{i}\right) \\
S_{2}=\sum_{i=1}^{n} \operatorname{Re}\left(X_{i}\right) \operatorname{Im}\left(Y_{i}\right)-\operatorname{Im}\left(X_{i}\right) \operatorname{Re}\left(Y_{i}\right) \\
S_{3}=\sum_{i=1}^{n}\left\|X_{i}\right\|^{2} \\
S_{4}=\sum_{i=1}^{n}\left\|Y_{i}\right\|^{2}
\end{gathered}
$$

\subsection{An Application Example}

Flutter is a typical dynamic aeroelastic instability phenomenon. It is induced by a self-excited physical mechanism that the vibrating aerodynamic surface absorbs energy from surrounded air flow. When the airspeed is lower, it will behave 
in a damped motion after perturbed. However, as it is up to some critical airspeed, the vibration amplitude will increase sometimes wildly, hence flutter occurs and always leads to a disaster. Flutter solution is mainly a stability analysis procedure. Firstly, the aeroelastic equation of motion is established, usually in frequency domain, where the unsteady aerodynamics is computed by panel methods, e.g. doublet lattice method (DLM) at subsonic range. Then followed is to solve the eigen roots of the flutter equation. Most of the root would be complex. The flutter occurs as the one of the roots' real part changes from negative to positive.

An example of T-tail structure is given, and its generalized mass, stiffness and aerodynamic matrix are extracted from the standard flutter solution sequence of NASTRAN'DMAP language. According to the eigenvalue solution and the eigenvector for the eigenvalue tracking, home-made program of P-K method is used to compute the flutter analysis. The $g \sim V$ and $\omega \sim V$ diagrams are illustrated:

As shown in Fig.1 and Fig.2, the aeroelastic flutter speed of the horizontal tail is $275 \mathrm{~m} / \mathrm{s}$, while that of the vertical tail is $335 \mathrm{~m} / \mathrm{s}$.

\section{Remark}

1) The numerical simulation demonstrations that the above method for flutter solution by use of eigenvalues and eigenvectors is proved to be a more reliable and robust flutter analysis than the standard P-K method.

2) Compared with the other eigenvalue tracking, this method using the eigenvector to determine the corresponding eigenvalue largely simplifies the computational and iterative process. In engineering, it is a practical and efficient method in frequency domain.

\section{References}

CAO Zhihao, ZHANG Yude, and LI Ruixia. (1979). Matrix Computation and solutions of Equation, High Education Publishing House, pp.177-194.

FU Jianhu, CHEN Gangming, and Nie Yufeng. (2001). Principle of Numerical Analysis, Science Publishing House, pp.236-237, 248-251.

MSC.Nastran Version 68, Aeroelastic Analysis User's Guide, pp. 32-40.

ZHAO Yonghui. (2007). Aeroelastics and Control, Science Publishing House, 2007, pp. 280-282.

Louw H van Zyl. (1992). Use of Eigenvectors in the Solution of the Flutter Equation, J. AIRCRAFT, VOL. 30, NO. 4, pp.553-554.

David Eller. (2009). Flutter Equation as a Piecewise Quadratic Eigenvalue Problem, J. AIRCRAFT, Vol. 46 , No. 3.

Larry Huttsell, Dave Schuster, John Volk, Joe Giesing, Mike Love. (2001). Evaluation of Computational Aeroelasticity Codes for Loads and Flutter, $A I A A$.

Eli Livne. (2003). Future of Airplane Aeroelasticity, J. AIRCRAFT, Vol. 40, No. 6.

David M. Schuster, Danny D. Liu, Lawrence J. Huttsell. (2003). Computational Aeroelasticity: Success, Progress, Challenge, AIAA.

St. Louis. (1999). A New Non-Iterative P-K Match Point Flutter Solution, AIAA.

I. E. Garrick and Wilmer H. Reed III. (1981). Historical Development of Aircraft Flutter, J. AIRCRAFT, VOL. 18, NO. 11.

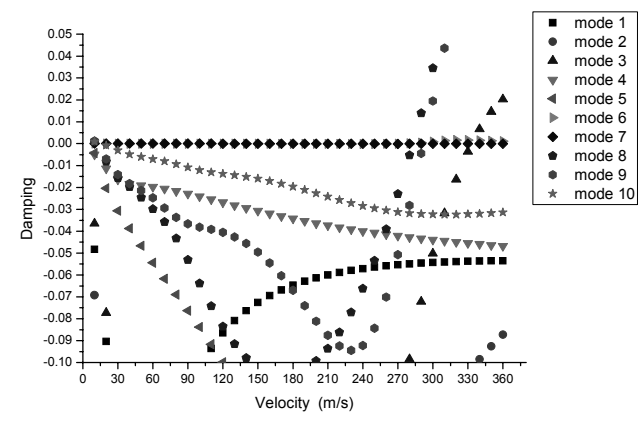

Figure 1. V-g Curve

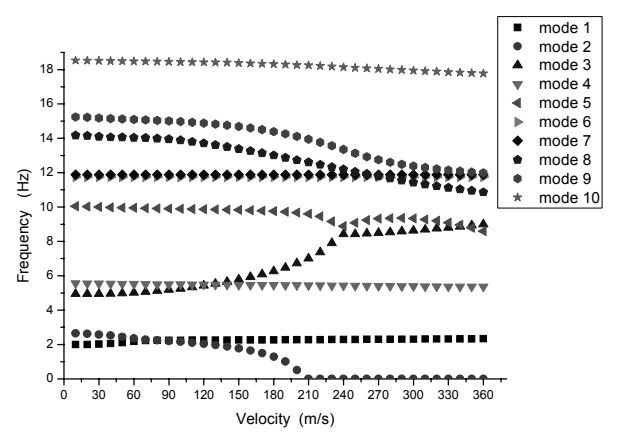

Figure 2. V-f Curve 